\title{
The Cortisol to Cortisone Ratio during Cardiac Catheterisation in Sows
}

\author{
Hana Skarlandtová', Marie Bičíková ${ }^{2}$ Petr Neužil ${ }^{3}$, Mikuláš Mlček', \\ Vladimír Hrachovina ${ }^{1}$,Tomáš Svoboda', Eva Medová', Jaroslav Kudlička', \\ Alena Dohnalová ${ }^{1}$, Štěpán Havránek ${ }^{4}$, Hana Kazihnítková ${ }^{2}$, \\ Ludmila Máčová ${ }^{2}$, Eva Vařejková ${ }^{1}$, Otomar Kittnar ${ }^{1}$ \\ ${ }^{1}$ Institute of Physiology, First Faculty of Medicine, Charles University in Prague, \\ Prague, Czech Republic; \\ ${ }^{2}$ Institute of Endocrinology, Prague, Czech Republic; \\ ${ }^{3}$ Department of Cardiology, Na Homolce Hospital, Prague, Czech Republic; \\ ${ }^{4} 2^{\text {nd }}$ Department of Medicine - Department of Cardiovascular Medicine, \\ First Faculty of Medicine, Charles University in Prague and General University \\ Hospital in Prague, Prague, Czech Republic \\ Received August 8, 2015 ; Accepted November 16, 2015.
}

Key words: Stress - Stress hormones - Cortisol/cortisone ratio Heart catheterisation - Sow

Abstract: A possible effect of mini-invasive heart intervention on a response of hypothalamo-pituitary-adrenal stress axis and conversion of cortisone to cortisol were studied. We have analysed two stress markers levels (cortisol, cortisone) and cortisol/cortisone ratio in 25 sows using minimally invasive heart catheterisation as the stress factor. The values of studied parameters were assessed in four periods of the experiment: (1) the baseline level on the day before intervention, (2) after the introduction of anaesthesia, (3) after conducting tissue stimulation or ablation, and (4) after the end of the catheterisation. For statistical analyses we used the nonparametric Friedman test for four dependent samples (including all four stages of the operation) or three dependent samples (influence of operation only, baseline level was excluded). Statistically significant differences in both Friedman tests were found for cortisol and for cortisone. We have found the highest level of cortisol/ cortisone ratio in unstressed conditions, then it decreased to the minimal level at

This study was supported by grants PRVOUK-P35/LF1/5 and the project MZ CR for conceptual development of research organization 00023761 (Institute of Endocrinology, Prague, Czech Republic).

Mailing Address: Prof. Otomar Kittnar, MD., PhD., Institute of Physiology, First Faculty of Medicine, Charles University in Prague, Albertov 5, 12800 Prague 2, Czech Republic; Phone: +420 224968 430; Fax: +420 224918 816; e-mail: otomar.kittnar@If1.cuni.cz 
the end of the intervention. We have concluded that cortisol levels are blunted by the influence of anaesthesia after its administration, and therefore decrease back to the baseline at the end of the operation.

\section{Introduction}

Stress and stress marker detection has been extensively discussed for many years in both human and veterinary medicine. Stimuli affecting the homeostasis of organism are called stressors and arise from many different origins e.g. ecological (acute environmental changes, absence of nutrition or shelter, temperature variations), sociobiological (unstable social hierarchy), health (infection, injury, surgery), transport and many others. Stressors trigger a stress response a complex of physiological, endocrinal, metabolic and behavioural reactions protecting the organism from the injurious effect (Schreiber, 1985; Greenberg et al., 2002; Möstl and Palme, 2002). In our presented study we have studied the stress response of young sows to an invasive heart catheterisation which was supposed to be a stressor. In order to evaluate the stress response of hypothalamo-pituitaryadrenal axis concentration of the adrenal cortex steroid (cortisol, cortisone) was determined as well as the cortisol/cortisone ratio.

Cortisol's molecular structure is lipophilic, allowing the unbound cortisol to enter freely the target cells through the cell membrane into the cytoplasm where it is bound to specific receptors. The cortisol-receptor complex then enters the nucleus and identifies glucocorticoid response elements (GREs), special palindromic DNA sequences, binds to them, and then acts as a transcription modulator (Seckl, 1997).

Cortisone is a steroid hormone also produced by the adrenal cortex. This hormone is characterised by its possession of a keto-group on C-11 (cortisol has hydroxyl group on C-11) and cannot be bound to cytoplasmatic receptors. Cortisone functions as a reserve pool of cortisol, providing more cortisol when needed (e.g. in stress response). Two isoenzymes of 11 $\beta$-hydroxysteroid dehydrogenase (11 $\beta-H S D)$ act as important regulation factors for the conversion of cortisone to cortisol (11 $\beta-H S D 1)$ and conversely cortisol to cortisone

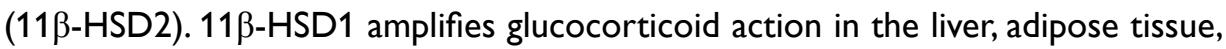
inflammatory cells, and vasculature, providing a therapeutic target for inhibition in type 2 diabetes. $11 \beta-H S D 2$ limits cortisol action, and thereby facilitates aldosterone action in the distal nephron and a few other sites (Kilgour et al., 2015).

The aim of the presented study was to determine stress marker levels including cortisol/cortisone ratio in each period of the experiment - we compared the level obtained at the breeding farm (under non-stress conditions) with levels obtained during the heart catheterisation experiment (potential stress events). Our second aim was to determine, if there are any differences in stress markers levels during minimally invasive heart intervention itself (the first blood collection was excluded). 
We hope our findings could help improve elective cardiac procedures to minimise their effect on human patients or animal recipients.

\section{Material and Methods}

For this study, serum concentrations of two adrenal activity markers (cortisol, cortisone) were determined in sows undergoing elective heart catheterisation. Although it was only minimally invasive surgery, this provided a stress overload event. Marker levels were measured during four well defined periods of the experiment (details were published elsewhere - Skarlandtová et al., 2012, and they are shortly described in section "Blood collection") in order to evaluate any changes in their concentrations. Our aim was to determine if elective minimally invasive intervention has an influence on the hypothalamo-pituitary-adrenal (HPA) axis and consequently on the activity of the adrenal cortex.

The experiment was performed in accordance with Czech law and corresponding EU regulations and was approved by the Institutional Animal Care and Use Committee.

\section{Animals}

Twenty-five four month old sows (Sus scrofa domestica) were used in the experiment, from the crossbreed Landrace $\times$ Large White, details of breeding, housing, etc. of animals were described in our previous publication (Skarlandtová et al., 2012). The sows were in prepubertal age; therefore we can exclude the estrous cycle influence on the stress marker levels.

\section{Experiment}

The effect of heart catheterisation on stress marker levels in blood serum was tested. Changes in blood serum concentrations of cortisol and cortisone were determined.

\section{Heart catheterisation}

Heart catheterisation was performed following the standard catheterization procedure (through arteria and vena femoralis using a 7-9F sheet). The catheterizations were carried out within the frame of electrophysiological projects, where cardiac stimulation or conducting tissue electrical radiofrequency ablation was performed. In all tested animals two markers were assessed in the blood serum: cortisol and cortisone.

\section{Anaesthesia and medication}

Stresnil $(5 \mathrm{mg} / \mathrm{kg})$, atropin $(0.05 \mathrm{mg} / \mathrm{kg})$ and narcetan $(14 \mathrm{mg} / \mathrm{kg})$ were used via intramuscular injection for pre-medication and sedation. An 18G or 20G IV cannula was inserted into the marginal ear vein to obtain intravenous (IV) access. Intravenous anaesthetic introduction was initiated using a propofol $(2 \mathrm{mg} / \mathrm{kg})$ and 
morphine $(0.2 \mathrm{mg} / \mathrm{kg})$ bolus. Intubation under direct laryngoscopic control was performed with 7 or $7.5 \mathrm{~mm}$ orotracheal tubes, depending on the size of the sow. Anaesthesia was maintained with a propofol $(4 \mathrm{mg} / \mathrm{kg} / \mathrm{h}) \mathrm{IV}$ infusion, and as an analgesic, a morphine $(0.2 \mathrm{mg} / \mathrm{kg})$ IV bolus was administered every hour.Ventilation was sustained at an average volume of 8 to $10 \mathrm{ml} / \mathrm{kg}$ and respiratory rates 15 per minute. No inhalation anaesthesia was used.

During the IV anaesthesia a continuous monitoring of mean arterial pressure (MAP), heartbeat rate $(\mathrm{HR}), \mathrm{O}_{2}$ saturation $\left(\mathrm{SO}_{2}\right)$ and exhalated capnometry $\left(\mathrm{PCO}_{2}\right)$ was observed on a multiparameter bed-side monitor.

\section{Blood collection}

Blood was collected from the jugular vein during each of the four defined periods of the experiment. The first (1) was collected at the farm, in non-stress domestic conditions (control sample, baseline stress marker levels), other samples were collected 10 minutes after the presumed stress situation: a second sample

(2) 10 minutes after intubation and the introduction to anaesthesia, a third sample

(3) 10 minutes after cardio stimulation or conducting tissue ablation and the last

(4) at the end of the intervention, before the animal was sacrificed. Blood samples $(10 \mathrm{ml})$ were collected in $10 \mathrm{ml}$ serum Vacutainer system tubes (BDVacutainer, SSt II Advance), and after a 30 minute incubation at room temperature were centrifuged $(2000 \times \mathrm{g})$ for 15 minutes and then serum was stored at $-20^{\circ} \mathrm{C}$ until later analysis. The whole experiment can be divided into two sections: the first period (non-stress conditions, baseline reference sample) and the second, surgery section (the second, third and fourth periods of the experiment) (Figure 1).

\section{Laboratory analyses}

Cortisol and cortisone were measured using the method published elsewhere (Šimůnková et al., 2008). In brief, the serum samples were twice extracted and then the hormones separated using a high performance liquid chromatography (HPLC)

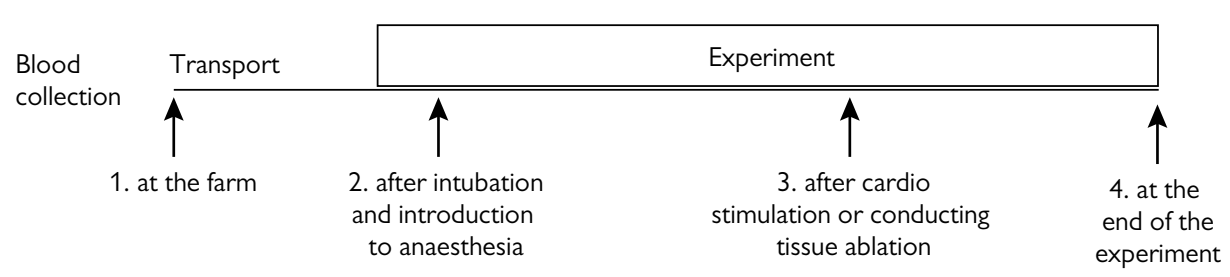

Figure 1 -The blood collection system. Arrows indicate the blood sampling in four defined parts of the experiment. For the first time, sows were blood sampled at the breeding farm, in non-stress conditions, after twenty-four hours were sows transported to the experimental laboratory, and then were blood sampled after intubation and introduction to anaesthesia (second sample), after cardiostimulation or conducting tissue ablation and the last, at the end of the catheterisation. 
system from Dionex Softron (Germering, Germany). HPLC separation was carried out with reverse phase EC 250/4 NUCLEOSIL ${ }^{\circledR}$ 100-5 C18 column (MACHEREYNAGEL, Düren, Germany), and to avoid possible column contamination the Phenomenex SecurityGuard system with cartridge C18 (Phenomenex, Torrance, CA) was used. Merck (Darmstadt, Germany) solvents were used as the mobile phase for HPLC. Cortisol and cortisone concentrations in the serum were determined according to a calibration curve using UV/VIS detection.

\section{Statistics}

As our data did not have a standard Gaussian distribution, non-parametric statistical methods were used to analyse the differences in stress marker levels within the experiment. For testing difference in stress markers levels within the experiment non-parametric Friedman test was used. Friedman test was also calculated for determining cortisol/cortisone ratio for four and three dependent samples.

\section{Results}

Elementary statistical data was calculated for the measured markers (cortisol, cortisone) for each period of the experiment (1-4). A wide variance in measured data was demonstrated, indicating substantial inter-individual differences in the assessed marker levels; for illustration, box and whisker plots (Figures 2-4) are shown for each marker. Medians (for exclusion outlier values) were noted and discussed, and the results shown separately for each marker.

\section{Cortisol}

As we expected, the serum cortisol concentration was the lowest $(148.35 \mathrm{nmol} / \mathrm{l})$ in the non-stress sample at the farm. Therefore we can regard the samples from the first period of the experiment as the baseline level. The cortisol concentration

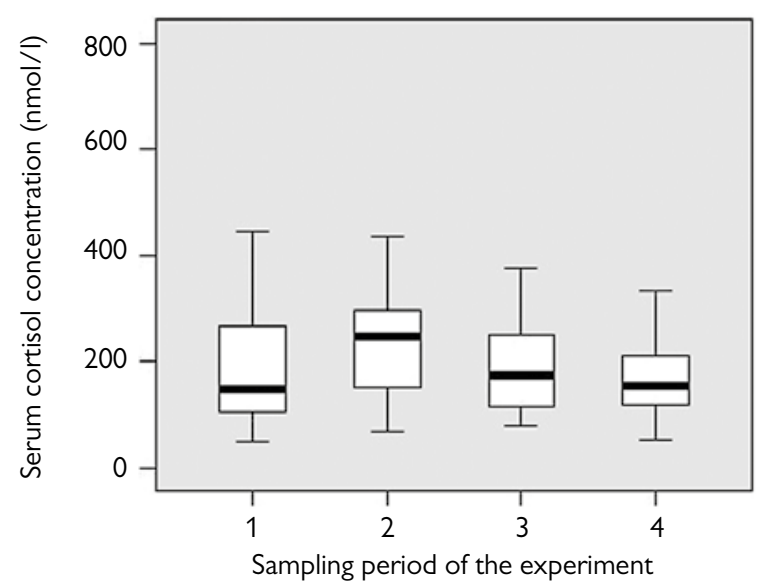

Figure 2 - Box and whisker plot: Serum cortisol concentrations during the four sampling periods of the experiment. There are $50 \%$ of measured values of cortisol concentration in the box, the median is marked as a bold line in the box, whiskers are $25 \%$ measured values. 

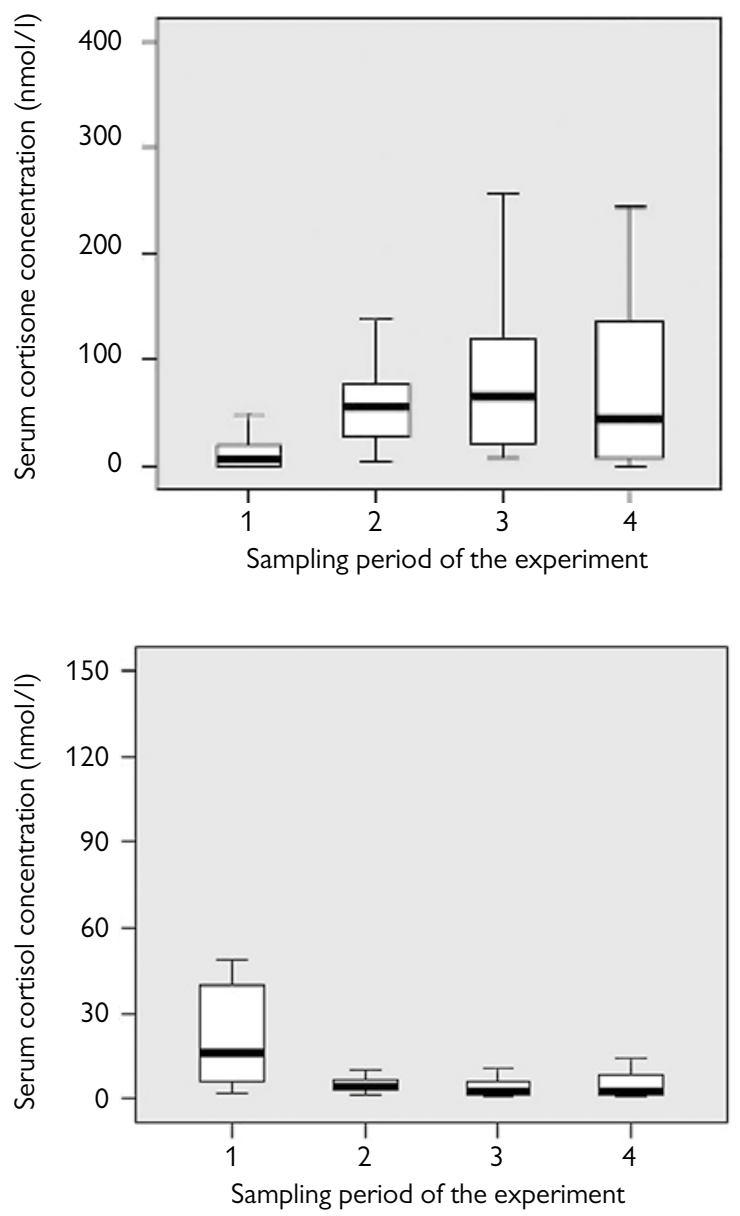

Figure 3 - Box and whisker plot: Serum cortisone concentrations during the four sampling periods of the experiment. There are $50 \%$ of measured values of cortisone concentration in the box, the median is marked as a bold line in the box, whiskers are $25 \%$ measured values.
Figure 4 - Box and whisker plot: Serum cortisol to cortisone ratio during the four sampling periods of the experiment. There are $50 \%$ of measured values of cortisone concentration in the box, the median is marked as a bold line in the box, whiskers are $25 \%$ measured values.

then increased to the highest level $(246.41 \mathrm{nmol} / \mathrm{l})$ during the second period of the experiment (after the introduction anaesthesia). During the third and fourth periods of the experiment, cortisol levels decreased to concentrations close to the baseline $(175.42 \mathrm{nmol} / \mathrm{l}$ and $154.30 \mathrm{nmol} / /$ respectively).

The statistics (Friedman test) were significant $(p<0.05)$ in both tests - for all four sampled periods of the experiment, and for the three stages excluding the first one. This suggests that the surgery section itself also had an important influence on the levels of cortisol.

\section{Cortisone}

Similar to the cortisol median levels, the concentration of cortisone was the lowest during the first period of the experiment $(6.19 \mathrm{nmol} / \mathrm{l})$. Levels increased during the second and third periods (the highest level was recorded during the 
third sampling time; $66.12 \mathrm{nmol} / \mathrm{l})$. The cortisone level then decreased at the end of the intervention, but was still seven times higher than the baseline $(44.70 \mathrm{nmol} / \mathrm{l})$. Statistics for all periods of the experiment were significant $(p<0.001)$, and, if we exclude the first stage of the experiment, statistics were $p<0.05$. This means that the cortisone levels statistically changed in all periods of the experiment, and also for the three stages of heart catheterisation when viewed separately from the non-stress (baseline) period.

\section{Cortisol/cortisone ratio}

Cortisol/cortisone ratio was calculated for each pig separately, median, minimum and maximum for each period of the experiment was determined. Median was the highest for the first period of the experiment (non-stress conditions) (15.95), then rapidly decreased and the lowest value was determined at the end of the surgery (2.51) (Table 1).

Friedman test for this ratio was statistically significant only for surgery periods of experiment compared to the baseline level under unstressed conditions $(p<0.001)$.

\section{Discussion}

Our study was focused on the HPA (hypothalamo-pituitary-adrenal) axis activity, but not on that of the SAM (sympatho-adreno-medullar) axis. The first and the most important reason was the fact that catecholamines (unlike glucocorticoids) are released in a few seconds following the stress stimulus, and the design of our experiment technically disallowed the blood collection in this very short time. Moreover it is unable to assess catecholamines and glucocorticoids (and other tested markers) in the same blood sample and two blood collections at every stage of the experiment could cause two problems: 1 ) the volume of the collected blood and 2) possible affection of the second sample results by the preceding blood collection.

Anaesthesia influence on adrenal hormones secretion Anaesthesia is used to minimize the traumatic effect of surgical procedures. It is well known that anaesthesia blunts stress response through the suppression of

\section{Table 1 - Ratio of cortisol/cortisol concentrations}

\begin{tabular}{lrrrr}
\hline $\begin{array}{l}\text { Sampling period } \\
\text { of experiment }\end{array}$ & Median & Minimum & Maximum & P \\
\hline 1 & 15.95 & 1.71 & 144.75 & \\
2 & 4.48 & 1.17 & 32.65 & $<0.001$ \\
3 & 2.61 & 0.44 & 17.37 & \\
4 & 2.51 & 0.73 & 20.38 & \\
\hline
\end{tabular}

${ }_{1}^{1}$ sampling period of the experiment: 1 ) the baseline level at non-stress conditions at home farm; 2 ) after intubation and introduction to anaesthesia; 3 ) after cardiac stimulation or conducting tissue ablation; 4) at the end of experiment 
the secretion of stress hormones (catecholamines and glucocorticoids). There are divergent types of anaesthesia with different suppression rates of stress response - balanced (inhalation) and total intravenous anaesthesia (TIVA). Many studies have found lower stress marker levels in TIVA (a combination of propofol and an opioid as an analgesic component) compared to balanced anaesthesia (a combination of inhalation gases e.g. sevoflurane, isoflurane, enflurane, etc. and an opioid) (Schricker et al., 2000; Ledowski et al., 2005; Ihn et al., 2009; Kostopanagiotou et al., 2010; Marana et al., 2010). TIVA, consisting of a combination of propofol and morphine, was used in our experiment (for details see in section "Anaesthesia and medication"). Propofol is lipophilic weak acid with voltage-gated ion L-calcium channels in heart influence. It improves decreased sympathetic activity alpha and beta adrenergic receptors (Krzych et al., 2009). Combination of propofol and morphine (or other opioid) decreasing catecholamines and glucocorticoid released to blood (Fragen et al., 1987; Schricker et al., 1999, 2000; Ihn et al., 2009).

The propofol/opioid combination suppresses HPA activity at each level. That is, the suppression of the production of the corticotrophin releasing hormone $(\mathrm{CRH})$, adrenocorticotropin hormone (ACTH) and the glucocorticoids. This could be caused by a synergic propofol-opioid reaction on the hypothalamic receptors that suppress noxious afferent stimuli and then suppress $\mathrm{CRH}$ release (due to the increase in the GABA receptor inhibitor concentration) (Kostopanagiotou et al., 2010; Marana et al., 2010).

Many authors (e.g. Van Hemelrijck et al., 1995; Han et al., 2012; Offinger et al., 2012) found decreased cortisol levels 45 minutes after the introduction of anaesthesia, which corresponds with our results. Stress steroid levels were lower in comparison to non-stress ones (e.g. Fragen et al., 1987; Schricker et al., 1999, 2000; Ihn et al., 2009). In our experiment, the results for cortisol concentration corresponded to referenced authors, with the highest levels determined in the second period of the experiment i.e. a very short time after the introduction to anaesthesia, then during the third and fourth periods the levels decreased approximately back to the baseline concentration. In comparison, cortisone concentrations increased during the second period of the experiment and were higher compared with the baseline levels. We attribute this to the balance stress reaction of these steroids.

\section{Surgical procedure}

In this experiment the minimally invasive surgical procedure of heart catheterization was used. A number of previous studies found no difference in elevated cortisol levels between invasive open surgery and minimally invasive procedures in pigs (Mansour et al., 1992; Bessler et al., 1994; Burpee et al., 2002; Margulis et al., 2005; Matsumoto et al., 2005; Duchene et al., 2008). These findings could mean that even minimal intervention can cause a rise in cortisol 
levels. However, stress response to surgery could be modulated by some other parameters, e.g. type of anaesthesia (mentioned above), handling, etc. Contrastingly, experimental measurements in human patients have found some differences in stress response for the same type of the laparoscopic intervention using a slightly different surgical approach (Han et al., 2012).

Our findings suggest that the most stressful stage of the heart catheterization is during its very beginning. This fact should be considered in invasive animal experiments but it would be very difficult to transfer this experience to human cardiac interventions particularly because of standard sedative premedication of human patients.

\section{Cortisol and cortisone}

Basic levels of cortisol in our group of experimental animals differ from some data reported previously (e.g. Perremans et al., 2001). The difference can be explained by many factors: different environmental conditions of live, procedure of cannulation, different genetic groups of animals, and big interindividual differences among individual animals. This was one of reasons why we had to measure our own basic levels of examined hormones and did not rely on literature data.

Serum cortisone concentration in our experiment was at the lowest level during the first period of the experiment, then it increased to the highest levels during the third period, and during the fourth period it slightly decreased. This corresponds with the cortisol level, which was found to be at the highest concentration after exposure to anaesthesia and then it decreased. We assume that the higher levels of cortisone were caused as a result of the higher activity of the 11ß-HSD2 isoenzyme (the crucial regulation factor of cortisol/cortisone levels), in this way balancing the action of the cortisol.

\section{Cortisone and cortisol/cortisone ratio}

Cortisone could be considered to be an inactive form of cortisol, but, on the other hand, it represents cortisol's reserve pool in the time when more cortisol is needed (e.g. in the stress response). The serum cortisol/cortisone ratio isoenzyme $11 \beta$-hydroxysteroid dehydrogenase $(11 \beta-H S D)$ balanced. The activity of these isoformes is crucial in cortisol/cortisone levels in blood serum. The mainly mechanisms in this role of these isoenzymes is presumably cortisol inactivation by $11 \beta-H S D 2$ in kidneys (higher activity caused cortisol inactivation) and 11 $\beta-H S D 1$ in the liver (cortisone restoration) (Vogeser et al., 2003).

\section{Conclusion}

In our experiment cortisol, cortisone and cortisol/cortisone levels were determined during four stages of the heart catheterisation of young sows. We separately calculated Friedman tests for each marker during the four defined periods of the experiment; these tests were statistically significant for all markers. 
The lowest concentration levels for both markers were measured during the baseline, non-stress conditions. Therefore, we can conclude that the conditions at sows' home farm are non-stressful. From the baseline, the serum concentrations of both markers then increased to their highest levels; for cortisol during the second period and for cortisone during the third one. We can assume that the adrenal secretion of cortisol occurs in response to the most stressful conditions before the exposure to intravenous anaesthesia, and then the anaesthesia minimizes the stress response. The other marker, cortisone, acts as a balanced system against the traumatic effects of stress. We have found the highest level of cortisol/cortisone ratio in unstressed conditions, then it decreased to minimally level at the end of the intervention, it could be due to anaesthesia minimizing the stress response and cortisol levels decreased after exposure the anaesthesia and cortisone levels were higher against baseline.

Acknowledgements:The authors thank to Bortelová J., Červenková J., Ehrlichová A., Kypta K. and Vavrikiková T. for their technical support and kind help during the experiments at the Cardiac Electrophysiology Experimental Laboratory (Na Homolce Hospital and First Faculty of Medicine, Charles University in Prague). Special thanks belong to C. Johnson for language help and proofreading the text.

\section{References}

Bessler, M., Whelan, R. L., Halverson, A., Treat, M. R., Nowygrod, R. (1994) Is immune function better preserved after laparoscopic versus open colon resection? Surg. Endosc. 8, 881-883.

Burpee, S. E., Kurian, M., Murakame, Y., Benevides, S., Gagner, M. (2002) The metabolic and immune response to laparoscopic versus open liver resection. Surg. Endosc. 16, 899-904.

Duchene, D. A., Gallagher, B. L., Ratliff, T. L., Winfield, H. N. (2008) Systemic and cell-specific immune response to laparoscopic and open nephrectomy in porcine model. J. Endourol. 22, 113-120.

Fragen, R. J., Weiss, H.W., Molteni, A. (1987) The effect of propofol on adrenocortical steroidogenesis: a comparative study with etomidate and thiopental. Anesthesiology 66, 839-842.

Greenberg, N., Carr, J. A., Summers, C. H. (2002) Ethological cause and consequences of the stress response. Integr. Comp. Biol. 42, 508-516.

Han, C., Ding, Z., Fan, J., Sun, J., Qian, Y. (2012) Comparison of the stress response in patients undergoing gynecological laparoscopic surgery using carbon dioxide pneumoperitoneum or abdominal wall-lifting methods. J. Laparoendosc. Adv. Surg. Tech. A 22, 330-335.

Ihn, C. H., Joo, J. D., Choi, J.W., Kim, D.W., Jeon, Y. S., Kim, Y. S., Jung, H. S., Kwon, S. Y. (2009) Comparison of stress hormone response, interleukin- 6 and anaesthetic characteristics of two anaesthetic techniques: volatile induction and maintenance of anaesthesia using sevoflurane versus total intravenous anaesthesia using propofol and remifentanil.J. Int. Med. Res. 7, 1760-1771.

Kilgour, A. H. M., Semple, S., Marshall, I., Andrews, P., Andrew, R., Walker, B. R. (2015) 11ß-hydroxysteroid dehydrogenase activity in the brain does not contribute to systemic interconversion of cortisol and cortisone in healthy men. J. Clin. Endocrinol. Metab. 100, 483-489.

Kostopanagiotou, G., Kalimeris, K., Christodoulaki, K., Nastos, C., Papoutsidakis, N., Dima, C., Chrelias, C., Pandazi, A., Mourouzis, I., Pantos, C. (2010) The differential impact of volatile and intravenous anaesthetics on stress response in the swine. Hormones 9, 67-75. 
Krzych, L. J., Szurlej, D., Bochenek, A. (2009) Rationale for propofol use in cardiac surgery. J. Cardiothorac. Vasc. Anesth. 23, 878-885.

Ledowski, T., Bein, B., Hanss, R., Paris, A., Fudickar,W., Scholz, J., Tonner, P. H. (2005) Neuroendocrine stress response and heart rate variability: a comparison of total intravenous versus balanced anesthesia. Anesth. Analg. 101, 1700-1705.

Mansour, M. A., Stiegmann, G.V., Yamamoto, M., Berguer, R. (1992) Neuroendocrine stress response after minimally invasive surgery in pigs. Surg. Endosc. 6, 294-297.

Marana, E., Colicci, S., Meo, F., Marana, R., Proietti, R. (2010) Neuroendocrine stress response in gynecological laparoscopy: TIVA with propofol versus sevoflurane anesthesia.J. Clin. Anesth. 22, 250-255.

Margulis, V., Matsumoto, E. D., Tunc, L., Taylor, G., Duchenne, D., Cadeddu, J. A. (2005) Effect of warmed, humidified insufflation gas and anti-inflammatory agents on cytokine response to laparoscopic nephrectomy: porcine model. J. Urol. 174, 1452-1456.

Matsumoto, E. D., Margulis, V., Tunc, L., Taylor, G. D., Duchene, D., Johnson, D. B., Pearle, M. S., Cadeddu, J. A. (2005) Cytokine response to surgical stress: comparison of pure laparoscopic, hand-assisted laparoscopic, and open nephrectomy. J. Endourol. 19, 1140-1145.

Möstl, E., Palme, R. (2002) Hormones as indicators of stress. Domest. Anim. Endocrinol. 23, 67-74.

Offinger, J., Meyer, H., Fischer, J., Kästner, S. B., Piechotta, M., Rehage, J. (2012) Comparison of isoflurane inhalation anaesthesia, injection anaesthesia and high volume caudal epidural anaesthesia for umbilical surgery in calves; metabolic, endocrine and cardiopulmonary effects. Vet. Anaesth. Analg. 39, 123-136.

Perremans, S., Randall, J. M., Rombouts, G., Decuypere, E., Geers, R. (2001) Effect of whole-body vibration in the vertical axis on cortisol and adrenocorticotropic hormone levels in piglets. J. Anim. Sci. 79, 975-981.

Schreiber, V. (1985) Stressors. In: Stress. Pathophysiology - Endocrinology - Clinics. Schreiber, V., pp. 18-21, Avicenum, Prague. (in Czech)

Schricker, T., Klubien, K., Carli, F. (1999) The independent effect of propofol anesthesia on whole body protein metabolism in humans. Anesthesiology 90, 1636-1642.

Schricker, T., Carli, F., Schreiber, M., Wachter, U., Geisser, W., Lattermann, R., Georgieff, M. (2000) Propofol/ sufentanil anesthesia suppresses the metabolic and endocrine response during, not after, lower abdominal surgery. Anesth. Analg. 90, 450-455.

Seckl, J. R. (1997) 11beta-hydroxysteroid dehydrogenase in the brain: a novel regulator of glucocorticoid action? Front. Neuroendocrinol. 18, 49-99.

Šimůnková, K., Stárka, L., Hill, M., Křiž, L., Hampl, R., Vondra, K. (2008) Comparison of total and salivary cortisol in a low-dose ACTH (Synacthen) test: Influence of three-month oral contraceptives administration to healthy women. Physiol. Res. 57, 193-199.

Skarlandtová, H., Bičíková, M., Neužil, P., Mlček, M., Hrachovina, V., Svoboda, T., Medová, E., Kudlička, J., Dohnalová, A., Havránek, Š., Kazihnítková, H., Máčová, L., Vařejková, E., Kittnar, O. (2012) Might cardiac catheterization influence diurnal rhythm of the steroid stress hormones secretion? Physiol. Res. 61, 25-34.

Van Hemelrijck, J., Weekers, F., Van Aken, H., Bouillon, R., Heyns, W. (1995) Propofol anesthesia does not inhibit stimulation of cortisol synthesis. Anesth. Analg. 80, 573-576.

Vogeser, M., Groetzner, J., Küpper, C., Briegel, J. (2003) The serum cortisol:cortisone ratio in the postoperative acute-phase response. Horm. Res. 59, 293-296. 